\title{
The First Integral Method to the Nonlinear Schrodinger Equations in Higher Dimensions
}

\author{
Shoukry Ibrahim Atia El-Ganaini ${ }^{1,2}$ \\ ${ }^{1}$ Mathematics Department, Faculty of Science, Damanhour University, Bahira 22514, Egypt \\ ${ }^{2}$ Mathematics Department, Faculty of Science and Humanity Studies at Al-Quwaiaiah, Shaqra University, \\ Al-Quwaiaiah 11971, Saudi Arabia \\ Correspondence should be addressed to Shoukry Ibrahim Atia El-Ganaini; ganaini5533@hotmail.com
}

Received 19 October 2012; Accepted 30 December 2012

Academic Editor: Elena Litsyn

Copyright (c) 2013 Shoukry Ibrahim Atia El-Ganaini. This is an open access article distributed under the Creative Commons Attribution License, which permits unrestricted use, distribution, and reproduction in any medium, provided the original work is properly cited.

The first integral method introduced by Feng is adopted for solving some important nonlinear partial differential equations, including the $(2+1)$-dimensional hyperbolic nonlinear Schrodinger (HNLS) equation, the generalized nonlinear Schrodinger (GNLS) equation with a source, and the higher-order nonlinear Schrodinger equation in nonlinear optical fibers. This method provides polynomial first integrals for autonomous planar systems. Through the established first integrals, exact traveling wave solutions are formally derived in a concise manner.

\section{Introduction}

It is well known that nonlinear complex physical phenomena are related to nonlinear partial differential equations (NLPDEs) which are involved in many fields from physics to biology, chemistry, mechanics, etc. As mathematical models of the phenomena, the investigation of exact solutions of NLPDEs will help us to understand these phenomena better. Many effective methods for obtaining exact solutions of NLPDEs have been established and developed, such as the Lie point symmetries method [1], the exp-function method $[2,3]$, the sine-cosine method $[4,5]$, the extended tanh-coth method $[6,7]$, the projective Riccati equation method $[8,9]$, and so on.

The first integral method was first proposed by Feng in [10] in solving Burgers-KdV equation which is based on the ring theory of commutative algebra. Recently, this useful method has been widely used by many such as in [11-21] and by the references therein. In Section 2, we have described this method for finding exact travelling wave solutions of nonlinear evolution equations. In Section 3, we have illustrated this method in detail with the (2+1)-dimensional hyperbolic nonlinear Schrodinger (HNLS) equation, the generalized nonlinear Schrodinger (GNLS) equation with a source, and the higher-order nonlinear Schrodinger equation in nonlinear optical fibers. In Section 4, we have given some conclusions.

\section{The First Integral Method}

Consider a general nonlinear PDE in the form

$$
P\left(u, u_{t}, u_{x}, u_{x x}, u_{t t}, u_{x t}, u_{x x x}, \ldots\right)=0,
$$

where $P$ is a polynomial in its arguments.

Raslan in [22] has summarized the first integral method in the following steps.

Step 1. Using a wave variable $\xi=x-c t+\varepsilon$, where $\varepsilon$ is an arbitrary constant, (1) can be written in the following nonlinear ordinary differential equation (ODE):

$$
Q\left(U, U^{\prime}, U^{\prime \prime}, U^{\prime \prime \prime}, \ldots\right)=0,
$$

where the prime denotes the derivation with respect to $\xi$.

Step 2. Assume that the solution of ODE (2) can be written as

$$
u(x, t)=u(\xi) .
$$


Step 3. We introduced new independent variables

$$
X(\xi)=u(\xi), \quad Y=u_{\xi}(\xi),
$$

which leads a system of nonlinear ODEs

$$
\begin{gathered}
X_{\xi}(\xi)=Y(\xi), \\
Y_{\xi}(\xi)=F(X(\xi), Y(\xi)) .
\end{gathered}
$$

Step 4. According to the qualitative theory of ODEs [23], if we can find the integrals to (5a) and (5b) under the same conditions, then the general solution to (5a) and (5b) can be found directly. However, in general, it is really difficult to realize this even for one first integral, because for a given plane autonomous system, there is no systematic theory that can tell us how to find its first integrals, nor is there a logical way for telling us what these first integrals are.

We will apply the division theorem to obtain one first integral to (5a) and (5b) which reduces (2) to a first-order integrable ODE.

An exact solution to (1) is then obtained by solving this equation.

Let us now recall the division theorem for two variables in the complex domain $C(w, z)$.

Theorem 1 (division theorem). Suppose that $P(w, z)$ and $Q(w, z)$ are polynomials in $C(w, z)$, and $P(w, z)$ is irreducible in $C(w, z)$. If $Q(w, z)$ vanishes at all zero points of $P(w, z)$, then there exists a polynomial $G(w, z)$ in $C(w, z)$ such that

$$
Q(w, z)=P(w, z) G(w, z) .
$$

The division theorem follows immediately from the Hilbert-Nullstellensatz Theorem [24].

Theorem 2 (Hilbert-Nullstellensatz theorem). Let $k$ be a field and $L$ an algebraic closure of $k$.

(1) Every ideal $\gamma$ of $k\left[X_{1}, \ldots, X_{n}\right]$ not containing 1 admits at least one zero in $L^{n}$.

(2) Let $x=\left(x_{1}, \ldots, x_{n}\right), y=\left(y_{1}, \ldots, y_{n}\right)$ be two elements of $L^{n}$; for the set of polynomials of $k\left[X_{1}, \ldots, X_{n}\right]$ zero at $x$ to be identical with the set of polynomials of $k\left[X_{1}, \ldots, X_{n}\right]$ zero at $y$ it is necessary and sufficient that there exists a $k$-automorphism $s$ of $L$ such that $y_{i}=s\left(x_{i}\right)$ for $1 \leq i \leq n$.

(3) For an ideal $\alpha$ of $k\left[X_{1}, \ldots, X_{n}\right]$ to be maximal, it is necessary and sufficient that there exists an $x$ in $L^{n}$ such that $\alpha$ is the set of polynomials of $k\left[X_{1}, \ldots, X_{n}\right]$ to be zero at $x$.

(4) For a polynomial $Q$ of $k\left[X_{1}, \ldots, X_{n}\right]$ to be zero on the set of zeros in $L^{n}$ of an ideal $\gamma$ of $k\left[X_{1}, \ldots, X_{n}\right]$, it is necessary and sufficient that there exists an integer $m>$ 0 such that $Q^{m} \in \gamma$.

\section{Applications}

In this section, we have investigated three NPDEs using the first integral method for the first time.
3.1. The (2+1)-Dimensional Hyperbolic Nonlinear Schrodinger Equation. Let us consider the (2+1)-dimensional hyperbolic nonlinear Schrodinger (HNLS) equation [25] which reads

$$
i u_{t}+\frac{1}{2} u_{x x}-\frac{1}{2} u_{y y}+|u|^{2} u=0
$$

where $x$ is dimensionless variable, $y$ is the propagation coordinate, $i=\sqrt{-1}$, and $t$ is the time. The above equation can be derived from optics [26] and large-scale Rossby waves [27]. Various types of NLS or HNLS equations describing time and space evolutions of slowly varying envelopes have wide applications in various branches of physics $[28,29]$.

By considering the transformations $u(x, y, t)=$ $\varphi(\xi) \exp (i \eta)$, and the wave variable $\xi=x+a y-c t+\varsigma$, $\eta=m x+n y+w t+\varepsilon$, where, $\varsigma$, and $\varepsilon$ are arbitrary constants, (7) changes into a system of ordinary differential equations as follows:

$$
\left(c^{2}-1\right) \varphi^{\prime \prime}(\xi)=2 \varphi^{3}(\xi)+\left(w^{2}-2 n-(a+c w)^{2}\right) \varphi(\xi),
$$

where prime denotes the derivative with respect to the same variable $\xi$.

Using (4) and (5a) and (5b), we can get

$$
\begin{aligned}
& X^{\prime}(\xi)=Y(\xi), \\
& Y^{\prime}(\xi)=\left(\frac{2}{c^{2}-1}\right) X^{3}(\xi)+\left(\frac{w^{2}-2 n-(a+c w)^{2}}{c^{2}-1}\right) X(\xi) .
\end{aligned}
$$

According to the first integral method, we suppose that $X(\xi)$ and $Y(\xi)$ are nontrivial solutions of $(9 a)$ and $(9 b)$ and $P(X, Y)=\sum_{i=0}^{m} a_{i}(X) Y^{i}$ is an irreducible polynomial in the complex domain $C[X, Y]$ such that

$$
P[X(\xi), Y(\xi)]=\sum_{i=0}^{m} a_{i}(X(\xi)) Y^{i}(\xi)=0
$$

where $a_{i}(X),(i=0,1,2, \ldots, m)$ are polynomials of $X$ and $a_{m}(X) \neq 0$.

Equation (10) is called the first integral to (9a) and (9b). Due to the division theorem, there exists a polynomial $h(X)+$ $g(X) Y$ in the complex domain $C[X, Y]$ such that

$$
\begin{aligned}
\frac{d P}{d \xi} & =\frac{\partial P}{\partial X} \frac{d X}{d \xi}+\frac{\partial P}{\partial Y} \frac{d Y}{d \xi} \\
& =[h(X)+g(X) Y] \sum_{i=0}^{m} a_{i}(X) Y^{i}
\end{aligned}
$$

Here, we have considered two different cases, assuming that $m=1$ and $m=2$ in (10). 
Case 1. Suppose that $m=1$, by equating the coefficients of $Y^{i}(i=2,1,0)$ on both sides of (11), we have

$$
\begin{gathered}
a_{1}^{\prime}(X)=g(X) a_{1}(X), \\
a_{0}^{\prime}(X)=h(X) a_{1}(X)+g(X) a_{0}(X), \\
a_{1}(X)\left(\left(\frac{2}{c^{2}-1}\right) X^{3}+\left(\frac{w^{2}-2 n-(a+c w)^{2}}{c^{2}-1}\right) X\right) \\
=h(X) a_{0}(X) .
\end{gathered}
$$

Since $a_{i}(X)(i=0,1)$ are polynomials, then from (12a) we have deduced that $a_{1}(X)$ is constant and $g(X)=0$. For simplicity, we have $a_{1}(X)=1$.

Balancing the degrees of $h(X)$ and $a_{0}(X)$, we have concluded that $\operatorname{deg}(h(X))=1$ only. Suppose that $h(X)=$ $A X+B$ and $A \neq 0$, we find $a_{0}(X)$

$$
a_{0}(X)=\frac{A}{2} X^{2}+B X+D
$$

where $D$ is an arbitrary integration constant.

Substituting $a_{0}(X), a_{1}(X)$, and $h(X)$ for (12c) and setting all the coefficients of powers $X$ to be zero, we have obtained a system of nonlinear algebraic equations and by solving it, we have obtained

$$
\begin{gathered}
D=\mp \frac{1}{2} \sqrt{\frac{1}{-1+c^{2}}}[2 n+(a+(-1+c) w)(a+w+c w)], \\
B=0, \quad A= \pm \sqrt{2} \sqrt{-\frac{1}{1-c}-\frac{1}{1+c}} .
\end{gathered}
$$

Using the conditions (14) in (10), we obtain

$$
\begin{aligned}
Y(\xi)=\mp \frac{\sqrt{2}}{2} \sqrt{-\frac{1}{1-c}}-\frac{1}{1+c} X^{2}(\xi) \\
\pm \frac{1}{2} \sqrt{\frac{1}{-1+c^{2}}}[2 n+(a+(-1+c) w) \\
\times(a+w+c w)],
\end{aligned}
$$

respectively.

Combining (15) with (9a), we have obtained the exact solutions to (9a) ad (9b). The exact traveling wave solutions to the $(2+1)$-dimensional hyperbolic nonlinear Schrodinger equation (7) can be written as

$$
\begin{aligned}
u_{1,2}(x, y, t)= & -\frac{1}{\sqrt{2}} \sqrt{-2 n-(a+(-1+c) w)(a+w+c w)} \\
\times \tan [ & \sqrt{-2 n-(a+(-1+c) w)(a+w+c w)} \\
\times & ( \pm \sqrt{-1+c}(x+a y-c t+\varsigma) \\
& \left.+2(-1+c) \sqrt{-1+c} \xi_{0}\right) \\
& \left.\times(\sqrt{2}(-1+c) \sqrt{-1+c})^{-1}\right] \\
& \times \exp [i(m x+n y+w t+\varepsilon)],
\end{aligned}
$$

respectively, where $\xi_{0}$ is an arbitrary integration constant.
Case 2. Assume that $m=2$, by equating the coefficients of $Y^{i}(i=3,2,1,0)$ on both sides of (11), we have

$$
\begin{gathered}
a_{2}^{\prime}(X)=g(X) a_{2}(X), \\
a_{1}^{\prime}(X)=h(X) a_{2}(X)+g(X) a_{1}(X), \\
a_{0}^{\prime}(X)+2 a_{2}(X)\left[\left(\frac{2}{c^{2}-1}\right) X^{3}+\left(\frac{w^{2}-2 n-(a+c w)^{2}}{c^{2}-1}\right) X\right] \\
=h(X) a_{1}(X)+g(X) a_{0}(X), \\
a_{1}(X)\left[\left(\frac{2}{c^{2}-1}\right) X^{3}+\left(\frac{w^{2}-2 n-(a+c w)^{2}}{c^{2}-1}\right) X\right] \\
=h(X) a_{0}(X) .
\end{gathered}
$$

Since, $a_{i}(X)(i=0,1,2)$ are polynomials, then from (17a) it can be deduced that $a_{2}(X)$ is a constant and $g(X)=0$. For simplicity, let us suppose that $a_{2}(X)=1$. Balancing the degrees of $h(X)$ and $a_{0}(X)$ it can be concluded that $\operatorname{deg}(h(X))=1$ only.

In this case, let us assume that $h(X)=A X+B$ and $A \neq 0$, then we find $a_{1}(X)$ and $a_{0}(X)$ as follows:

$$
\begin{aligned}
a_{1}(X)= & \left(\frac{A}{2}\right) X^{2}+B X+D, \\
a_{0}(X)= & \left(\frac{A^{2}}{8}-\frac{1}{c^{2}-1}\right) X^{4}+\frac{1}{2}(A B) X^{3} \\
& +\left(\frac{A D+B^{2}}{2}-\frac{w^{2}-2 n-(a+c w)^{2}}{c^{2}-1}\right) X^{2} \\
& +B D X+F,
\end{aligned}
$$

where $A, B, D$, and $F$ are arbitrary integration constants.

Substituting $a_{0}(X), a_{1}(X), a_{2}(X)$, and $h(X)$ for $(17 \mathrm{~d})$ and setting all the coefficients of powers $X$ to be zero, then we have obtained a system of nonlinear algebraic equations and by solving it, we get

$$
\begin{aligned}
& F=0, \quad D=0, \quad B=0, \\
& w=\frac{-a c-\sqrt{a^{2}+2 n-2 c^{2} n}}{-1+c^{2}}, \\
& A=\mp 2 \sqrt{2} \sqrt{-\frac{1}{1-c}-\frac{1}{1+c}}, \\
& F=0, \quad D=0, \quad B=0, \\
& w=\frac{-a c+\sqrt{a^{2}+2 n-2 c^{2} n}}{-1+c^{2}}, \\
& A=\mp 2 \sqrt{2} \sqrt{-\frac{1}{1-c}-\frac{1}{1+c}} .
\end{aligned}
$$

Using the conditions (19a) and (19b) in (10), we have obtained

$$
Y(\xi)= \pm \frac{1}{\sqrt{-1+c^{2}}} X^{2}(\xi) \text {. }
$$


Combining (20) with (9a) we have obtained the exact solutions to (9a) and (9b) and hence the exact traveling wave solutions to the $(2+1)$-dimensional hyperbolic nonlinear Schrodinger equation (7) can be written as

$$
\begin{aligned}
u_{3,4}(x, y, t) & \frac{\sqrt{-1+c} \sqrt{1+c}}{\mp(x+a y-c t+\varsigma)-\sqrt{-1+c} \sqrt{1+c}} \\
& \times \exp \left[i\left(m x+n y+\frac{-a c-\sqrt{a^{2}+2 n-2 c^{2} n}}{-1+c^{2}} t+\varepsilon\right)\right], \\
u_{5,6} & (x, y, t) \\
= & \frac{\mp(x+a y-c t+\varsigma)-\sqrt{-1+c} \sqrt{1+c}}{-1+c^{2}} \sqrt{1+c} \\
& \times \exp \left[i\left(m x+n y+\frac{-a c+\sqrt{a^{2}+2 n-2 c^{2} n}}{-1} t+\varepsilon\right)\right] .
\end{aligned}
$$

Comparing these results with the results obtained in [1], it can be seen that the solutions here are new.

\subsection{The Generalized Nonlinear Schrodinger (GNLS) Equation} with a Source. Let us Consider the generalized nonlinear Schrodinger (GNLS) equation with a source [30, 31], in the form

$$
\begin{aligned}
i u_{t}+ & a u_{x x}+b u|u|^{2}+i c u_{x x x}+i d\left(u|u|^{2}\right)_{x} \\
& =k e^{i[\chi(\xi)-w t]},
\end{aligned}
$$

where $\xi=\alpha(x-v t)$ is a real function and $a, b, c, d, k, \alpha, v$, and $w$ are all real.

The GNLS equation (23) plays an important role in many nonlinear sciences. It arises as an asymptotic limit for a slowly varying dispersive wave envelope in a nonlinear medium. For example, its significant application in optical soliton communication plasma physics has been proved.

Furthermore, the GNLS equation enjoys many remarkable properties (e.g., bright and dark soliton solutions, Lax pair, Liouvile integrability, inverse scattering transformation, conservation laws, Backlund transformation, etc.).

We have considered a plane wave transformation in the form

$$
u(x, t)=\psi(\xi) e^{i[\chi(\xi)-w t]}
$$

where $\psi(\xi)$ is a real function. For convenience, let $\chi=\beta \xi+x_{0}$ where $\beta$ and $x_{0}$ are real constants and $\xi=\alpha(x-v t)+\varsigma$. Then by replacing (23) and its appropriate derivatives in (22) and separating the real and imaginary parts of the result, we have obtained the following two ordinary differential equations:

$$
\begin{aligned}
& c \alpha^{3} \psi^{\prime \prime \prime}+\left(-\alpha v+2 a \beta \alpha^{2}-3 c \alpha^{3} \beta^{2}\right) \psi^{\prime} \\
& \quad+3 d \alpha \psi^{2} \psi^{\prime}=0, \\
& \left(a \alpha^{2}-3 c \psi^{3} \beta\right) \psi^{\prime \prime}+\left(\alpha \beta v+w-a \alpha^{2} \beta^{2}+c \alpha^{3} \beta^{3}\right) \psi \\
& \quad+(b-d \alpha \beta) \psi^{3}-k=0 .
\end{aligned}
$$

Integrating (25) once, with respect to $\xi$, we have

$$
c \alpha^{2} \psi^{\prime \prime}(\xi)+\left(-v+2 a \alpha \beta-3 c \alpha^{2} \beta^{2}\right) \psi(\xi)-M=0,
$$

where $M$ is an arbitrary integration constant. Since the same function $\psi(\xi)$ satisfies (26) and (27), we have obtained the following constraint condition:

$$
\begin{aligned}
\frac{a \alpha^{2}-3 c \psi^{3} \beta}{c \alpha^{2}} & =\frac{\alpha \beta v+w-a \alpha^{2} \beta^{2}+c \alpha^{3} \beta^{3}}{-v+2 a \alpha \beta-3 c \alpha^{2} \beta^{2}} \\
& =\frac{b-d \alpha \beta}{d}=\frac{k}{M} .
\end{aligned}
$$

Using (4) and (5a) and (5b), we can get

$$
\begin{aligned}
X^{\prime}(\xi)= & Y(\xi) \\
Y^{\prime}(\xi)= & \left(-\frac{d}{c \alpha^{2}}\right) X^{3}(\xi) \\
& +\left(\frac{v}{c \alpha^{2}}-\frac{2 a \beta}{c \alpha}+3 \beta^{2}\right) X(\xi) \\
& +\frac{M}{c \alpha^{2}}
\end{aligned}
$$

According to the first integral method, we suppose that $X(\xi)$ and $Y(\xi)$ are nontrivial solutions of (29a) and (29b) and $P(X, Y)=\sum_{i=0}^{m} a_{i}(X) Y^{i}$ is an irreducible polynomial in the complex domain $C[X, Y]$ such that

$$
P[X(\xi), Y(\xi)]=\sum_{i=0}^{m} a_{i}(X(\xi)) Y(\xi)^{i}=0,
$$

where $a_{i}(X),(i=0,1,2, \ldots, m)$ are polynomials of $X$ and $a_{m}(X) \neq 0$.

Equation (30) is called the first integral to (29a) and (29b). Due to the division theorem, there exists a polynomial $h(X)+$ $g(X) Y$ in the complex domain $C[X, Y]$ such that

$$
\begin{aligned}
\frac{d P}{d \xi} & =\frac{\partial P}{\partial X} \frac{d X}{d \xi}+\frac{\partial P}{\partial Y} \frac{d P}{d \xi} \\
& =[h(X)+g(X) Y] \sum_{i=0}^{m} a_{i}(X) Y^{i}
\end{aligned}
$$

In this example, we have taken two different cases, assuming that $m=1$ and $m=2$ in (30). 
Case 3. Suppose that $m=1$, by equating the coefficients of $Y^{i}(i=2,1,0)$ on both sides of $(31)$, we have

$$
\begin{gathered}
a_{1}^{\prime}(X)=g(X) a_{1}(X), \\
a_{0}^{\prime}(X)=h(X) a_{1}(X)+g(X) a_{0}(X), \\
a_{1}(X)\left[\left[-\frac{d}{c \alpha^{2}}\right] X^{3}+\left[\frac{v}{c \alpha^{2}}-\frac{2 a \beta}{c \alpha}+3 \beta^{2}\right] X+\frac{M}{c \alpha^{2}}\right] \\
=h(X) a_{0}(X) .
\end{gathered}
$$

Since $a_{i}(X)(i=0,1)$ are polynomials, then from (32a) it can be deduced that $a_{1}(X)$ is constant and $g(X)=0$. For simplicity, it was taken $a_{1}(X)=1$.

Balancing the degrees of $h(X)$ and $a_{0}(X)$, it can be concluded that $\operatorname{deg}(h(X))=1$ only. Suppose that $h(X)=$ $A X+B$, and $A \neq 0$, then we find

$$
a_{0}(X)=\frac{A}{2} X^{2}+B X+D
$$

where $D$ is an arbitrary integration constant.

Substituting $a_{0}(X), a_{1}(X)$, and $h(X)$ in (32c) and setting all the coefficients of powers $X$ to be zero, we have obtained a system of nonlinear algebraic equations and by solving it, we obtain

$$
\begin{aligned}
& v=-i \sqrt{2} \sqrt{c} \sqrt{d} D \alpha+2 a \alpha \beta-3 c \alpha^{2} \beta^{2}, \\
& M=0, \quad A=-\frac{i \sqrt{2} \sqrt{d}}{\sqrt{c} \alpha}, \quad B=0, \\
& v=i \sqrt{2} \sqrt{c} \sqrt{d} D \alpha+2 a \alpha \beta-3 c \alpha^{2} \beta^{2}, \\
& M=0, \quad A=\frac{i \sqrt{2} \sqrt{d}}{\sqrt{c} \alpha}, \quad B=0 .
\end{aligned}
$$

Using the conditions (34a) and (34b) in (30), we obtain

$$
Y(\xi)=\left( \pm \frac{i \sqrt{2} \sqrt{d}}{\sqrt{c} \alpha}\right) X^{2}(\xi)-D,
$$

respectively.

Combining (35) with (29a), the exact solutions to (29a) and (29b) were obtained and then the exact traveling wave solutions to the generalized nonlinear Schrodinger (GNLS) equation with a source (23) can be written as

$$
\begin{aligned}
u_{1}(x, t)= & i(-2)^{1 / 4} c^{1 / 4} \sqrt{D} \sqrt{\alpha} \\
& \times \tanh \left[(1+i) d^{1 / 4} \sqrt{D}\left(\alpha x-\alpha v t+\varsigma-2 \sqrt{c} \alpha \xi_{0}\right)\right. \\
& \left.\times\left(2^{3 / 4} c^{1 / 4} \sqrt{\alpha}\right)^{-1}\right] \times\left(d^{1 / 4}\right)^{-1} \\
& \times \exp [i(\beta\{\alpha x-\alpha v t+\varsigma\}-w t)] \\
v & =-i \sqrt{2} \sqrt{c} \sqrt{d} D \alpha+2 a \alpha \beta-3 c \alpha^{2} \beta^{2}
\end{aligned}
$$

$$
\begin{aligned}
u_{2}(x, t)= & i(-2)^{1 / 4} c^{1 / 4} \sqrt{D} \sqrt{\alpha} \\
& \times \tan \left[(1+i) d^{1 / 4} \sqrt{D}\left(\alpha x-\alpha v t+\varsigma-2 \sqrt{c} \alpha \xi_{0}\right)\right. \\
& \left.\times\left(2^{3 / 4} c^{1 / 4} \sqrt{\alpha}\right)^{-1}\right] \times\left(d^{1 / 4}\right)^{-1} \\
& \times \exp [i(\beta\{\alpha x-\alpha v t+\varsigma\}-w t)], \\
v & =i \sqrt{2} \sqrt{c} \sqrt{d} D \alpha+2 a \alpha \beta-3 c \alpha^{2} \beta^{2},
\end{aligned}
$$

where $\xi_{0}$ is an arbitrary integration constant.

Case 4. Suppose that $m=2$, by equating the coefficients of $Y^{i}(i=3,2,1,0)$ on both sides of $(31)$, we have

$$
\begin{gathered}
a_{2}^{\prime}(X)=g(X) a_{2}(X), \\
a_{1}^{\prime}(X)=h(X) a_{2}(X)+g(X) a_{1}(X), \\
a_{0}^{\prime}(X)+2 a_{2}(X)\left[\left[-\frac{d}{c \alpha^{2}}\right] X^{3}+\left[\frac{v}{c \alpha^{2}}-\frac{2 a \beta}{c \alpha}+3 \beta^{2}\right] X+\frac{M}{c \alpha^{2}}\right] \\
=h(X) a_{1}(X)+g(X) a_{0}(X), \\
a_{1}(X)\left[\left[-\frac{d}{c \alpha^{2}}\right] X^{3}+\left[\frac{v}{c \alpha^{2}}-\frac{2 a \beta}{c \alpha}+3 \beta^{2}\right] X+\frac{M}{c \alpha^{2}}\right] \\
=h(X) a_{0}(X) .
\end{gathered}
$$

Since $a_{i}(X)(i=0,1,2)$ are polynomials, then from (38a) it can be deduced that $a_{2}(X)$ is a constant and $g(X)=0$. For simplicity, we have taken $a_{2}(X)=1$. Balancing the degrees of $h(X)$ and $a_{0}(X)$ we have concluded that $\operatorname{deg}(h(X))=1$ only.

In this case, it was assumed that $h(X)=A X+B$ and $A \neq 0$; then we find $a_{1}(X)$ and $a_{0}(X)$ as follows:

$$
\begin{aligned}
a_{1}(X)= & \left(\frac{A}{2}\right) X^{2}+B X+D, \\
a_{0}(X)= & \left(\frac{A^{2}}{8}+\frac{d}{2 c \alpha^{2}}\right) X^{4}+\frac{1}{2}(A B) X^{3} \\
& +\left(\frac{A D+B^{2}}{2}-c \alpha^{2}+\frac{2 a \beta}{c \alpha}-3 \beta^{2}\right) X^{2} \\
& +\left(B D-\frac{2 M}{c \alpha^{2}}\right) X+F,
\end{aligned}
$$

where $A, B, D$, and $F$ are arbitrary integration constants.

Substituting $a_{0}(X), a_{1}(X), a_{2}(X)$, and $h(X)$ for $(38 \mathrm{~d})$ and setting all the coefficients of powers $X$ to be zero, a system of 
nonlinear algebraic equations was obtained and by solving it, we got

$$
\begin{gathered}
M=0, \quad v=\frac{1}{2}\left[-i \sqrt{2} \sqrt{c} \sqrt{d} D \alpha+4 a \alpha \beta-6 c \alpha^{2} \beta^{2}\right], \\
F=\frac{D^{2}}{4}, \quad A=-\frac{2 i \sqrt{2} \sqrt{d}}{\sqrt{c} \alpha}, \quad B=0, \\
M=0, \quad v=\frac{1}{2}\left[i \sqrt{2} \sqrt{c} \sqrt{d} D \alpha+4 a \alpha \beta-6 c \alpha^{2} \beta^{2}\right] \\
F=\frac{D^{2}}{4}, \quad A=\frac{2 i \sqrt{2} \sqrt{d}}{\sqrt{c} \alpha}, \quad B=0 .
\end{gathered}
$$

Using the conditions (40a) and (40b) in (30), we obtain

$$
Y(\xi)=\frac{ \pm i \sqrt{2} \sqrt{d} X^{2}(\xi)-\sqrt{c} D \alpha}{2 \sqrt{c} \alpha},
$$

respectively. Combining (41) with (29a) we have obtained the exact solutions to (29a) and (29b) and thus the exact traveling wave solutions to the generalized nonlinear Schrodinger (GNLS) equation with a source (23) can be written as

$$
\begin{aligned}
u_{3}(x, t)= & (-1)^{3 / 4} c^{1 / 4} \sqrt{D} \sqrt{\alpha} \\
& \times \tanh \left[\left(\frac{1}{2}+\frac{i}{2}\right) d^{1 / 4} \sqrt{D}\right. \\
& \times\left(\alpha x-\alpha v t+\varsigma-2 \sqrt{c} \alpha \xi_{0}\right) \\
& \left.\times\left(2^{1 / 4} c^{1 / 4} \sqrt{\alpha}\right)^{-1}\right] \times\left(2^{1 / 4} d^{1 / 4}\right)^{-1} \\
& \times \exp [i(\beta\{\alpha x-\alpha v t+\varsigma\}-w t)] \\
v= & \frac{1}{2} i \sqrt{2} \sqrt{c} \sqrt{d} D \alpha+2 a \alpha \beta-3 c \alpha^{2} \beta^{2} \\
u_{4}(x, t)= & (-1)^{3 / 4} c^{1 / 4} \sqrt{D} \sqrt{\alpha} \\
& \times \tan \left[(-1)^{1 / 4} d^{1 / 4}\right. \\
& \times \sqrt{D}\left(-\alpha x+\alpha v t+\varsigma+2 \sqrt{c} \alpha \xi_{0}\right) \\
v & \left.\times\left(2^{3 / 4} c^{1 / 4} \sqrt{\alpha}\right)^{-1}\right] \times\left(2^{1 / 4} d^{1 / 4}\right)^{-1} \\
& \times \exp [i(\beta\{\alpha x-\alpha v t+\varsigma\}-w t)] \\
1 & \\
& \\
& \\
&
\end{aligned}
$$

respectively, where $\xi_{0}$ is an arbitrary integration constant.

Equations (36)-(37) and (42)-(43) are new types of exact traveling wave solutions to the generalized nonlinear Schrodinger (GNLS) equation with a source (23). It could not be obtained by the methods presented in [32].
3.3. The Higher-Order Nonlinear Schrodinger Equation in Nonlinear Optical Fibers. The higher-order nonlinear Schrodinger equation describing propagation of ultrashort pulses in nonlinear optical fibers [33-39] reads

$$
\begin{aligned}
\psi_{z}= & i \alpha_{1} \psi_{t t}+i \alpha_{2} \psi|\psi|^{2}+\alpha_{3} \psi_{t t t} \\
& +\alpha_{4}\left(\psi|\psi|^{2}\right)_{t}+\alpha_{5} \psi\left(|\psi|^{2}\right)_{t}
\end{aligned}
$$

where $\psi$ is slowly varying envelope of the electric field, the subscripts $z$ and $t$ are the spatial and temporal partial derivative in retard time coordinates, and $\alpha_{1}, \alpha_{2}, \alpha_{3}, \alpha_{4}$, $\alpha_{5}$ are the real parameters related to the group velocity dispersion (GVD), self-phase modulation (SPM), third-order dispersion (TOD), and self-steepening and self-frequency shift arising from simulated Raman scattering, respectively. Some properties of the equation, as well as many versions of it have been studied [33-39]. Up to now, the bright, dark and the combined bright and dark solitary waves and periodic waves were found of (43) and its special case.

To seek traveling wave solutions of (44), we make the gauge transformation

$$
\begin{gathered}
\psi(z, t)=\varphi(\xi) \exp [i(k z-\omega t)] \\
\xi=\beta t-\lambda z+\varepsilon
\end{gathered}
$$

where $\beta, k, \omega, \lambda, \varepsilon$ are constants. Substituting (45) into (44) yields a complex ODE of $\varphi(\xi)$, the real and imaginary parts of which, respectively,

$$
\begin{gathered}
\left(\beta^{2} \alpha_{1}-3 \beta^{2} \alpha_{3} \omega\right) \varphi^{\prime \prime}+\left(\alpha_{3} \omega^{3}-\alpha_{1} \omega^{2}-k\right) \varphi \\
+\left(\alpha_{2}-\alpha_{4} \omega\right) \varphi^{3}=0, \\
\beta^{3} \alpha_{3} \varphi^{\prime \prime \prime}+\left(2 \beta \alpha_{1} \omega-3 \beta \alpha_{3} \omega^{2}+\lambda\right) \varphi^{\prime} \\
+\left(3 \beta \alpha_{4}+2 \beta \alpha_{5}\right) \varphi^{2} \varphi^{\prime}=0 .
\end{gathered}
$$

It is easy to see that (46) becomes an equation

$$
\varphi^{\prime \prime}+\frac{2 \beta \alpha_{1} \omega-3 \beta \alpha_{3} \omega^{2}+\lambda}{\beta^{3} \alpha_{3}} \varphi+\frac{3 \alpha_{4}+2 \alpha_{5}}{3 \beta^{2} \alpha_{3}} \varphi^{3}=0
$$

under the constraint conditions

$$
\begin{aligned}
\omega= & \frac{3 \alpha_{1} \alpha_{4}+2 \alpha_{1} \alpha_{5}-3 \alpha_{2} \alpha_{3}}{6 \alpha_{3}\left(\alpha_{4}+\alpha_{5}\right)}, \\
k= & \frac{1}{\alpha_{3}}\left[\frac{1}{\beta}\left(3 \alpha_{3} \omega-\alpha_{1}\right) \lambda-2 \omega\left(\alpha_{1}-2 \alpha_{3} \omega\right)^{2}\right] \\
= & \frac{\left(3 \alpha_{2} \alpha_{3}-3 \alpha_{1} \alpha_{4}-2 \alpha_{1} \alpha_{5}\right)\left(3 \alpha_{2} \alpha_{3}+\alpha_{1} \alpha_{5}\right)^{2}}{27 \alpha_{3}^{2}\left(\alpha_{4}+\alpha_{5}\right)^{3}} \\
& +\frac{\left(\alpha_{1} \alpha_{4}-3 \alpha_{2} \alpha_{3}\right) \lambda}{2 \beta \alpha_{3}\left(\alpha_{4}+\alpha_{5}\right)} .
\end{aligned}
$$


Using (4) and (5a) and (5b), we can get

$$
\begin{gathered}
X^{\prime}=Y, \\
Y^{\prime}=-\left(\frac{3 \alpha_{4}+2 \alpha_{5}}{3 \beta^{2} \alpha_{3}}\right) X^{3} \\
-\left(\frac{2 \beta \alpha_{1} \omega-3 \beta \alpha_{3} \omega^{2}+\lambda}{\beta^{3} \alpha_{3}}\right) X .
\end{gathered}
$$

According to the first integral method, we suppose that $X(\xi)$ and $Y(\xi)$ are nontrivial solutions of (49a) and (49b) and $P(X, Y)=\sum_{i=0}^{m} a_{i}(X) Y^{i}$ is an irreducible polynomial in the complex domain $C[X, Y]$ such that

$$
P[X(\xi), Y(\xi)]=\sum_{i=0}^{m} a_{i}(X(\xi)) Y(\xi)^{i}=0
$$

where $a_{i}(X),(i=0,1,2, \ldots, m)$ are polynomials of $X$ and $a_{m}(X) \neq 0$.

Equation (50) is called the first integral to (49a) and (49b) due to the division theorem, there exists a polynomial $h(X)+$ $g(X) Y$ in the complex domain $C[X, Y]$ such that

$$
\begin{aligned}
\frac{d P}{d \xi} & =\frac{\partial P}{\partial X} \frac{d X}{d \xi}+\frac{\partial P}{\partial Y} \frac{d P}{d \xi} \\
& =[h(X)+g(X) Y] \sum_{i=0}^{m} a_{i}(X) Y^{i}
\end{aligned}
$$

In this example, we take two different cases, assuming that $m=1$ and $m=2$ in (50).

Case 5. Suppose that $m=1$, by equating the coefficients of $Y^{i}(i=2,1,0)$ on both sides of $(51)$, we have

$$
\begin{gathered}
a_{1}^{\prime}(X)=g(X) a_{1}(X), \\
a_{0}^{\prime}(X)=h(X) a_{1}(X)+g(X) a_{0}(X), \\
a_{1}(X)\left[-\left(\frac{3 \alpha_{4}+2 \alpha_{5}}{3 \beta^{2} \alpha_{3}}\right) X^{3}-\left(\frac{2 \beta \alpha_{1} \omega-3 \beta \alpha_{3} \omega^{2}+\lambda}{\beta^{3} \alpha_{3}}\right) X\right] \\
=h(X) a_{0}(X) .
\end{gathered}
$$

Since $a_{i}(X)(i=0,1)$ are polynomials, then from (52a) it was deduced that $a_{1}(X)$ is constant and $g(X)=0$. For simplicity, take $a_{1}(X)=1$.

Balancing the degrees of $h(X)$ and $a_{0}(X)$, it was concluded that $\operatorname{deg}(h(X))=1$ only. Suppose that $h(X)=A X+B$ and $A \neq 0$, then we find

$$
a_{0}(X)=\frac{A}{2} X^{2}+B X+D
$$

where $D$ is an arbitrary integration constant.

Substituting $a_{0}(X), a_{1}(X)$, and $h(X)$ in (52c) and setting all the coefficients of powers $X$ to be zero, then we have obtained a system of nonlinear algebraic equations and by solving it, we obtain

$$
\begin{gathered}
D= \pm \frac{\sqrt{3 / 2}\left(\lambda+\beta \omega\left(2 \alpha_{1}-3 \omega \alpha_{3}\right)\right)}{\beta^{2} \sqrt{\alpha_{3}} \sqrt{-3 \alpha_{4}-2 \alpha_{5}}}, \\
B=0, \\
A=\mp \frac{\sqrt{2 / 3} \sqrt{-3 \alpha_{4}-2 \alpha_{5}}}{\beta \sqrt{\alpha_{3}}} .
\end{gathered}
$$

Using the conditions (54) in (50), we obtain

$$
\begin{aligned}
Y(\xi) & =\left( \pm \frac{\sqrt{2 / 3} \sqrt{-3 \alpha_{4}-2 \alpha_{5}}}{\beta \sqrt{\alpha_{3}}}\right) X^{2}(\xi) \\
& \mp \frac{\sqrt{3 / 2}\left(\lambda+\beta \omega\left(2 \alpha_{1}-3 \omega \alpha_{3}\right)\right)}{\beta^{2} \sqrt{\alpha_{3}} \sqrt{-3 \alpha_{4}-2 \alpha_{5}}},
\end{aligned}
$$

respectively.

Combining (55) with (49a), the exact solutions to (49a) and $(49 \mathrm{~b})$ were obtained and then the exact traveling wave solutions to the higher-order nonlinear Schrodinger equation in nonlinear optical fibers can be written as

$$
\begin{aligned}
& \psi_{1,2}(z, t) \\
&= \pm \frac{1}{\sqrt{\beta} p} \sqrt{3} q \\
& \times \tan \left[q\left((\beta t-\lambda z+\varepsilon) r \pm 2 \sqrt{3} \beta^{2} \xi_{0} \sqrt{\alpha_{3}} p^{2}\right)\right. \\
&\left.\times\left(2 \beta^{3 / 2} \sqrt{\alpha_{3}} p\right)^{-1}\right] \times \exp [i(k z-\omega t)],
\end{aligned}
$$

respectively, where

$$
\begin{gathered}
p=\sqrt{3 \alpha_{4}+2 \alpha_{5}}, \quad q=\sqrt{\lambda+\beta w\left(2 \alpha_{1}-3 w \alpha_{3}\right)}, \\
r=\sqrt{-6 \alpha_{4}-4 \alpha_{5}}, \quad p^{2}=3 \alpha_{4}+2 \alpha_{5},
\end{gathered}
$$

and $\xi_{0}$ is an arbitrary integration constant.

Case 6. Suppose that $m=2$, by equating the coefficients of $Y^{i}(i=3,2,1,0)$ on both sides of $(51)$, we have

$$
\begin{gathered}
a_{2}^{\prime}(X)=g(X) a_{2}(X), \\
a_{1}^{\prime}(X)=h(X) a_{2}(X)+g(X) a_{1}(X), \\
a_{0}^{\prime}(X)+2 a_{2}(X)\left[\left[-\frac{d}{c \alpha^{2}}\right] X^{3}+\left[\frac{v}{\alpha^{2}}-\frac{2 a \beta}{c \alpha}+3 \beta^{2}\right] X+\frac{M}{c \alpha^{2}}\right] \\
=h(X) a_{1}(X)+g(X) a_{0}(X), \\
a_{1}(X)\left[-\left(\frac{2 \beta \alpha_{1} \omega-3 \beta \alpha_{3} \omega^{2}+\lambda}{\beta^{3} \alpha_{3}}\right) X-\left(\frac{3 \alpha_{4}+2 \alpha_{5}}{3 \beta^{2} \alpha_{3}}\right) X^{3}\right] \\
=h(X) a_{0}(X) .
\end{gathered}
$$


Since $a_{i}(X)(i=0,1,2)$ are polynomials, then from (58a) it was deduced that $a_{2}(X)$ is a constant and $g(X)=0$. For simplicity, we have taken $a_{2}(X)=1$. Balancing the degrees of $h(X)$ and $a_{0}(X)$ we conclude that $\operatorname{deg}(h(X))=1$ only.

In this case, it was assumed that $h(X)=A X+B$ and $A \neq 0$; then we find $a_{1}(X)$ and $a_{0}(X)$ as follows:

$$
\begin{aligned}
a_{1}(X)= & \left(\frac{A}{2}\right) X^{2}+B X+D, \\
a_{0}(X)= & \left(\frac{A^{2}}{8}+\frac{3 \alpha_{4}+2 \alpha_{5}}{6 \beta^{2} \alpha_{3}}\right) X^{4}+\frac{1}{2}(A B) X^{3} \\
& +\left(\frac{A D+B^{2}}{2}+\frac{2 \beta \alpha_{1} \omega-3 \beta \alpha_{3} \omega^{2}+\lambda}{\beta^{3} \alpha_{3}}\right) X^{2} \\
& +B D X+F,
\end{aligned}
$$

where $A, B, D$, and $F$ are arbitrary integration constants.

Substituting $a_{0}(X), a_{1}(X), a_{2}(X)$, and $h(X)$ into (58d) and setting all the coefficients of powers $X$ to be zero, a system of nonlinear algebraic equations was obtained and by solving it, we get

$$
\begin{gathered}
\lambda=-2 \beta \omega \alpha_{1}+3 \beta \omega^{2} \alpha_{3}, \quad F=0, \quad B=0, \quad D=0, \\
A=\mp \frac{2 \sqrt{2 / 3} \sqrt{-3 \alpha_{4}-2 \alpha_{5}}}{\beta \sqrt{\alpha_{3}}}, \\
\lambda=-2 \beta \omega \alpha_{1}+3 \beta \omega^{2} \alpha_{3} \pm \frac{D \beta^{2} \sqrt{\alpha_{3}} \sqrt{-3 \alpha_{4}-2 \alpha_{5}}}{\sqrt{6}}, \\
F=\frac{D^{2}}{4}, \quad B=0, \quad A=\mp \frac{2 \sqrt{2 / 3} \sqrt{-3 \alpha_{4}-2 \alpha_{5}}}{\beta \sqrt{\alpha_{3}}} .
\end{gathered}
$$

Using the conditions (60a) in (50), we obtain

$$
Y(\xi)= \pm \frac{\sqrt{-3 \alpha_{4}-2 \alpha_{5}}}{\sqrt{6} \beta \sqrt{\alpha_{3}}} X^{2}(\xi),
$$

respectively. Combining (61) with (49a), the exact solutions to $(49 \mathrm{a})$ and (49b) were obtained and thus the exact traveling wave solutions to the higher-order nonlinear Schrodinger equation in nonlinear optical fibers (44) can be written as

$$
\begin{aligned}
& \psi_{3,4}(z, t) \\
& \begin{aligned}
= & \beta \sqrt{\alpha_{3}} \\
\times & \left(-6 \beta c_{1} \sqrt{\alpha_{3}}\right. \\
& \left.\mp \sqrt{6}\left(\beta t-\left(-2 \beta \omega \alpha_{1}+3 \beta \omega^{2} \alpha_{3}\right) z+\varepsilon\right) i p\right)^{-1} \\
\times & \exp [i(k z-\omega t)],
\end{aligned}
\end{aligned}
$$

respectively.
Similarly, in the case of (60b), from (50), we get

$$
Y(\xi)=-\frac{D}{2} \pm \frac{\sqrt{-3 \alpha_{4}-2 \alpha_{5}}}{\sqrt{6} \beta \sqrt{\alpha_{3}}} X^{2}(\xi),
$$

respectively. Combining (63) with (49a), the exact solutions to (49a) and (49b) were obtained and thus the exact traveling wave solutions to the higher-order nonlinear Schrodinger equation in nonlinear optical fibers (44) can be written as

$$
\begin{aligned}
& \psi_{5}(z, t)=(3 / 2)^{1 / 4} \sqrt{D} \sqrt{\beta} \alpha_{3}{ }^{1 / 4} p \\
& \times \tan [ \sqrt{D}\left((\beta t-\lambda z+\varepsilon)-6 \beta \xi_{0} \sqrt{\alpha_{3}}\right) p \\
&\left.\times\left(2^{3 / 4} 3^{1 / 4} \sqrt{\beta} \alpha_{3}{ }^{1 / 4}(-1)^{1 / 4} p^{1 / 2}\right)^{-1}\right] \\
& \times\left((-1)^{3 / 4} p^{3 / 2}\right)^{-1} \times \exp [i(k z-\omega t)], \\
& \lambda=-2 \beta \omega \alpha_{1}+3 \beta \omega^{2} \alpha_{3}+\frac{D \beta^{2} \sqrt{\alpha_{3}} \sqrt{-3 \alpha_{4}-2 \alpha_{5}}}{\sqrt{6}}
\end{aligned}
$$

$$
\begin{aligned}
& \psi_{6}(z, t)=(3 / 2)^{1 / 4} \sqrt{D} \sqrt{\beta} \alpha_{3}{ }^{1 / 4} \\
& \times p \tanh \left[\sqrt{D}\left((\beta t-\lambda z+\varepsilon)-6 \beta \xi_{0} \sqrt{\alpha_{3}}\right) p\right. \\
&\left.\times\left(2^{3 / 4} 3^{1 / 4} \sqrt{\beta} \alpha_{3}^{1 / 4}(-1)^{1 / 4} p^{1 / 2}\right)^{-1}\right] \\
& \times\left((-1)^{3 / 4} p^{3 / 2}\right)^{-1} \times \exp [i(k z-\omega t)], \\
& \lambda=-2 \beta \omega \alpha_{1}+3 \beta \omega^{2} \alpha_{3}-\frac{D \beta^{2} \sqrt{\alpha_{3}} \sqrt{-3 \alpha_{4}-2 \alpha_{5}}}{\sqrt{6}}
\end{aligned}
$$

respectively, where $p$ is as defined in (57) and $\xi_{0}$ is an arbitrary integration constant.

Comparing these results with Liu's results [39], it can be seen that the solutions here are new.

\section{Conclusion}

Searching for first integrals of nonlinear ODEs is one of the most important problems since they permit us to solve a nonlinear differential equation by quadratures. Applying the first integral method, which is based on the ring theory of commutative algebra, some new exact traveling wave solutions to the $(2+1)$-dimensional hyperbolic nonlinear Schrodinger (HNLS) equation, generalized nonlinear Schrodinger (GNLS) equation with a source and higher-order nonlinear Schrodinger equation in nonlinear optical fibers were established.

These solutions may be important for the explanation of some practical physical problems.

The first integral method described herein is not only efficient but also has the merit of being widely applicable. 
Therefore, this method can be applied to other nonlinear evolution equations and this will be done elsewhere.

\section{Acknowledgment}

The author would like to thank the referees for their useful comments which led to some improvements of the current paper.

\section{References}

[1] A.-L. Guo and J. Lin, "Exact solutions of $(2+1)$-dimensional HNLS equation," Communications in Theoretical Physics, vol. 54, no. 3, pp. 401-406, 2010.

[2] A.-M. Wazwaz, "Solitary wave solutions of the generalized shallow water wave (GSWW) equation by Hirotas method, tanhcoth method and Exp-function method," Applied Mathematics and Computation, vol. 202, no. 1, pp. 275-286, 2008.

[3] J.-H. He and L.-N. Zhang, "Generalized solitary solution and compacton-like solution of the Jaulent-Miodek equations using the Exp-function method," Physics Letters A, vol. 372, no. 7, pp. 1044-1047, 2008.

[4] A.-M. Wazwaz, "The tanh and the sine-cosine methods for a reliable treatment of the modified equal width equation and its variants," Communications in Nonlinear Science and Numerical Simulation, vol. 11, no. 2, pp. 148-160, 2006.

[5] A.-M. Wazwaz, "Solitary wave solutions for modified forms of Degasperis-Procesi and Camassa-Holm equations," Physics Letters A, vol. 352, no. 6, pp. 500-504, 2006.

[6] E. Fan, "Extended tanh-function method and its applications to nonlinear equations," Physics Letters A, vol. 277, no. 4-5, pp. 212-218, 2000.

[7] M. A. Abdou, "The extended tanh method and its applications for solving nonlinear physical models," Applied Mathematics and Computation, vol. 190, no. 1, pp. 988-996, 2007.

[8] R. Conte and M. Musette, "Link between solitary waves and projective Riccati equations," Journal of Physics A, vol. 25, no. 21, pp. 5609-5623, 1992.

[9] D. C. Lu and B. J. Hong, "New exact solutions for the (2+1)dimensional generalized Broer-Kaup system," Applied Mathematics and Computation, vol. 199, no. 2, pp. 572-580, 2008.

[10] Z. Feng, "The first-integral method to study the BurgersKorteweg-de Vries equation," Journal of Physics A, vol. 35, no. 2, pp. 343-349, 2002.

[11] Z.S. Feng and X. H. Wang, "The first integral method to the twodimensional Burgers-KdV equation," Physics Letters A, vol. 308, pp. 173-178, 2002.

[12] Z. Feng, S. Zheng, and D. Y. Gao, "Traveling wave solutions to a reaction-diffusion equation," Zeitschrift für Angewandte Mathematik und Physik, vol. 60, no. 4, pp. 756-773, 2009.

[13] Z. Feng and R. Knobel, "Traveling waves to a BurgersKorteweg-de Vries-type equation with higher-order nonlinearities," Journal of Mathematical Analysis and Applications, vol. 328, no. 2, pp. 1435-1450, 2007.

[14] K. R. Raslan, "The first integral method for solving some important nonlinear partial differential equations," Nonlinear Dynamics, vol. 53, no. 4, pp. 281-286, 2008.

[15] S. Abbasbandy and A. Shirzadi, "The first integral method for modified Benjamin-Bona-Mahony equation," Communications in Nonlinear Science and Numerical Simulation, vol. 15, no. 7, pp. 1759-1764, 2010.
[16] F. Tascan, A. Bekir, and M. Koparan, "Travelling wave solutions of nonlinear evolution equations by using the first integral method," Communications in Nonlinear Science and Numerical Simulation, vol. 14, no. 5, pp. 1810-1815, 2009.

[17] I. Aslan, "Exact and explicit solutions to nonlinear evolution equations using the division theorem," Applied Mathematics and Computation, vol. 217, no. 20, pp. 8134-8139, 2011.

[18] S. I. A. El-Ganaini, "Travelling wave solutions to the generalized Pochhammer-Chree (PC) equations using the first integral method," Mathematical Problems in Engineering, vol. 2011, Article ID 629760, 13 pages, 2011.

[19] M. F. El-Sabbagh and S. El-Ganaini, "New exact travelling wave solutions of the generalized Zakharov system via distinct methods," International Mathematical Forum, vol. 7, pp. 21912204, 2012.

[20] S. El-Ganaini, "Exact solutions of the equation of onedimensional motion of a pion meson particle in an atom using two different approaches," Advanced Studies in Theoretical Physics, vol. 6, no. 17-20, pp. 843-854, 2012.

[21] M. F. El-Sabbagh and S. I. El-Ganaini, "The first integral method and its applications to nonlinear equations," Applied Mathematical Sciences, vol. 6, no. 77-80, pp. 3893-3906, 2012.

[22] K. R. Raslan, "The first integral method for solving some important nonlinear partial differential equations," Nonlinear Dynamics, vol. 53, no. 4, pp. 281-286, 2008.

[23] T. R. Ding and C. Z. Li, Ordinary Differential Equations, Peking University Press, Peking, China, 1996.

[24] N. Bourbaki, Commutative Algebra, Addison-Wesley, Paris, France, 1972.

[25] S.P. Gorz, P.K. Ockaert, P. Emplit, and M. Haelterman, "Oscillatory neck instability of spatial bright solitons in hyperbolic systems," Physical Review Letters, vol. 102, no. 13, pp. 134101134104, 2009.

[26] S. P. Gorz and M. Haelterman, "Ultrafast transverse undulation of self-trapped laser beams," Optics Express, vol. 16, p. 16935, 2008.

[27] B. K. Tan and R. S. Wu, "Nonlinear Rossby waves and their interactions. I. Collision of envelope solitary Rossby waves," Science in China B, vol. 36, no. 11, pp. 1367-1380, 1993.

[28] X.-Y. Tang and P. K. Shukla, "Lie symmetry analysis of the quantum Zakharov equations," Physica Scripta, vol. 76, no. 6, pp. 665-668, 2007.

[29] B. Li, "A generalized sub-equation expansion method and its application to the nonlinear schrödinger equation in inhomogeneous optical fiber media," International Journal of Modern Physics C, vol. 18, no. 7, pp. 1187-1201, 2007.

[30] Z. Yan, "Envelope exact solutions for the generalized nonlinear Schrödinger equation with a source," Journal of Physics, vol. 39, no. 24, pp. L401-L406, 2006.

[31] M. A. Abdou, "New exact travelling wave solutions for the generalized nonlinear Schroedinger equation with a source," Chaos, Solitons \& Fractals, vol. 38, no. 4, pp. 949-955, 2008.

[32] M. Y. Moghaddam, A. Asgari, and H. Yazdani, "Exact travelling wave solutions for the generalized nonlinear Schrödinger (GNLS) equation with a source by extended tanh-coth, sinecosine and exp-function methods," Applied Mathematics and Computation, vol. 210, no. 2, pp. 422-435, 2009.

[33] Z. Y. Yan, "Generalized method and its application in the higher-order nonlinear Schrodinger equation in nonlinear optical fibres," Chaos, Solitons and Fractals, vol. 16, no. 5, pp. 759-766, 2003. 
[34] H. Y. Zhi, X. Q. Zhao, and H. Q. Zhang, "New expansion algorithm of three Riccati equations and its applications in nonlinear mathematical physics equations," Chinese Physics, vol. 14, no. 7, pp. 1296-1302, 2005.

[35] S. L. Palacios, A. Guinea, J. M. Fernández-Díaz, and R. D. Crespo, "Dark solitary waves in the nonlinear Schrödinger equation with third order dispersion, self-steepening, and selffrequency shift," Physical Review E, vol. 60, no. 1, pp. R45-R47, 1999.

[36] Z. H. Li, L. Li, H. P. Tian, and G. Zhou, "New types of solitary wave solutions for the higher order nonlinear Schrödinger equation," Physical Review Letters, vol. 84, no. 18, pp. 40964099, 2000.

[37] J. P. Tian, H. P. Tian, Z. H. Li, L. Kang, and G. Zhou, "An intermodulated solitary wave solution for the higher order nonlinear Schrödinger equation," Physica Scripta, vol. 67, no. 4, pp. 325$328,2003$.

[38] M. Gedalin, T. C. Scott, and Y. B. Band, "Optical solitary waves in the higher order nonlinear Schrödinger equation," Physical Review Letters, vol. 78, no. 3, pp. 448-451, 1997.

[39] C. Liu, "Exact solutions for the higher-order nonlinear Schördinger equation in nonlinear optical fibres," Chaos, Solitons and Fractals, vol. 23, no. 3, pp. 949-955, 2005. 


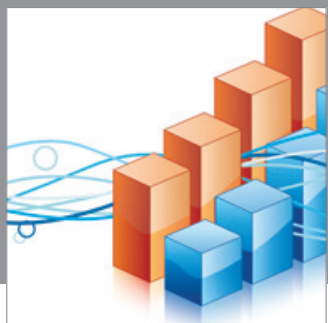

Advances in

Operations Research

mansans

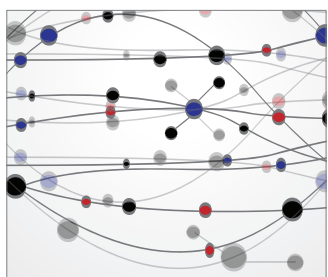

The Scientific World Journal
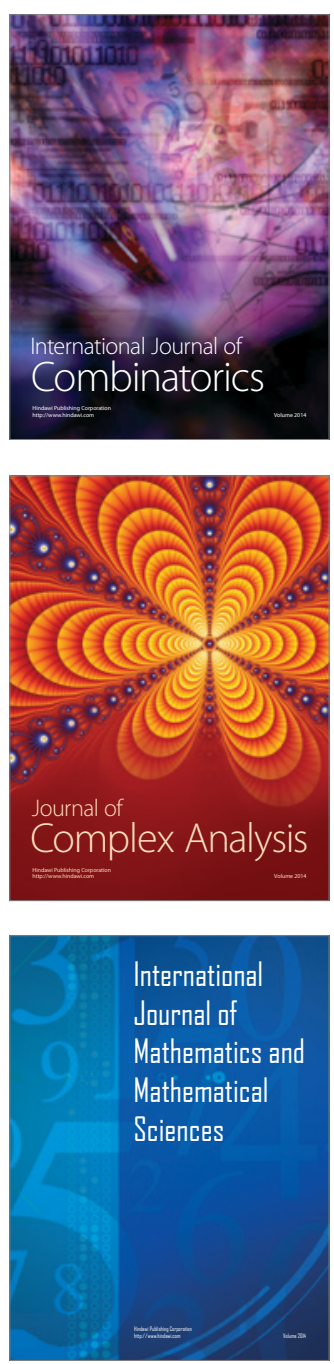
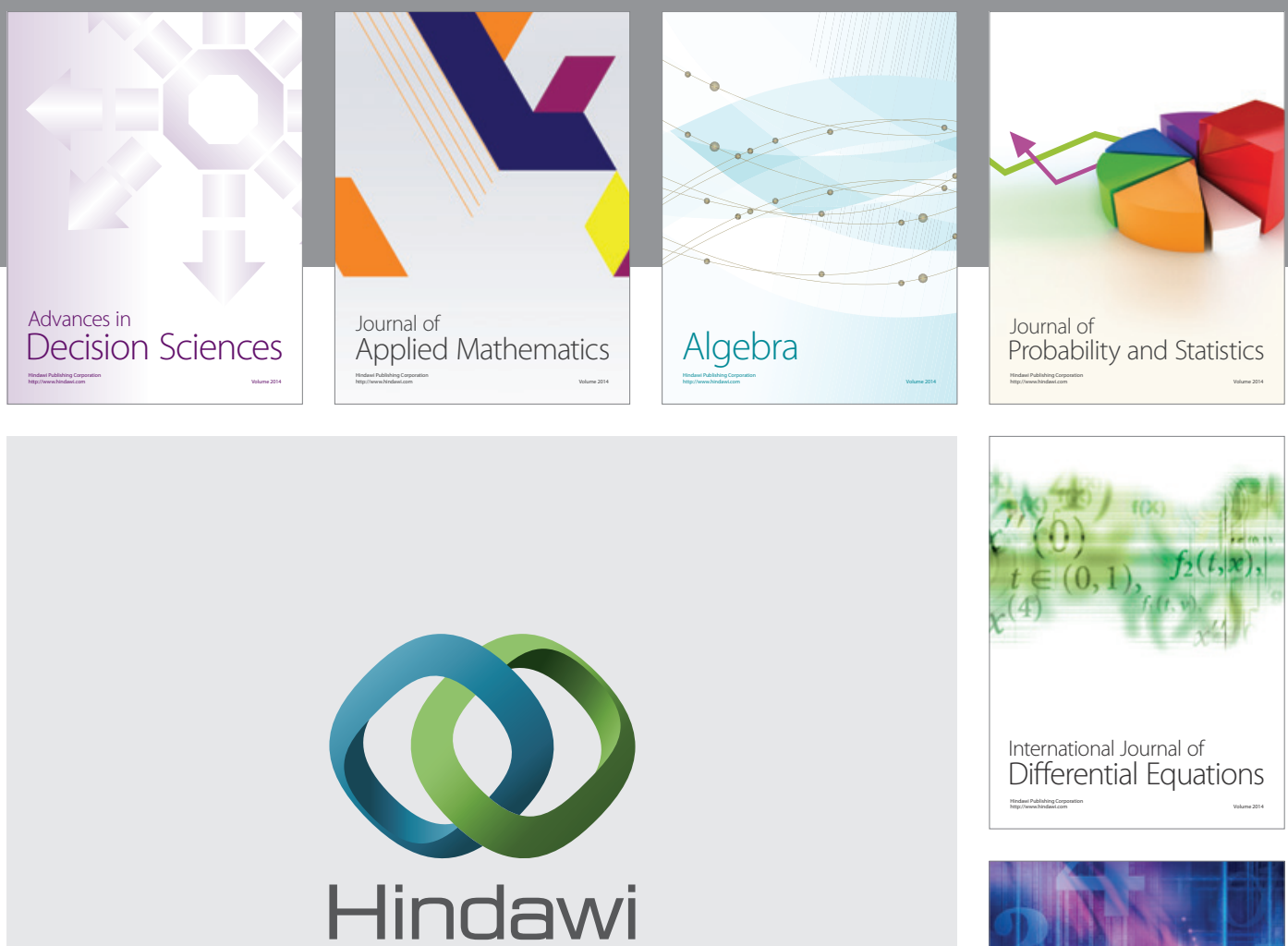

Submit your manuscripts at http://www.hindawi.com
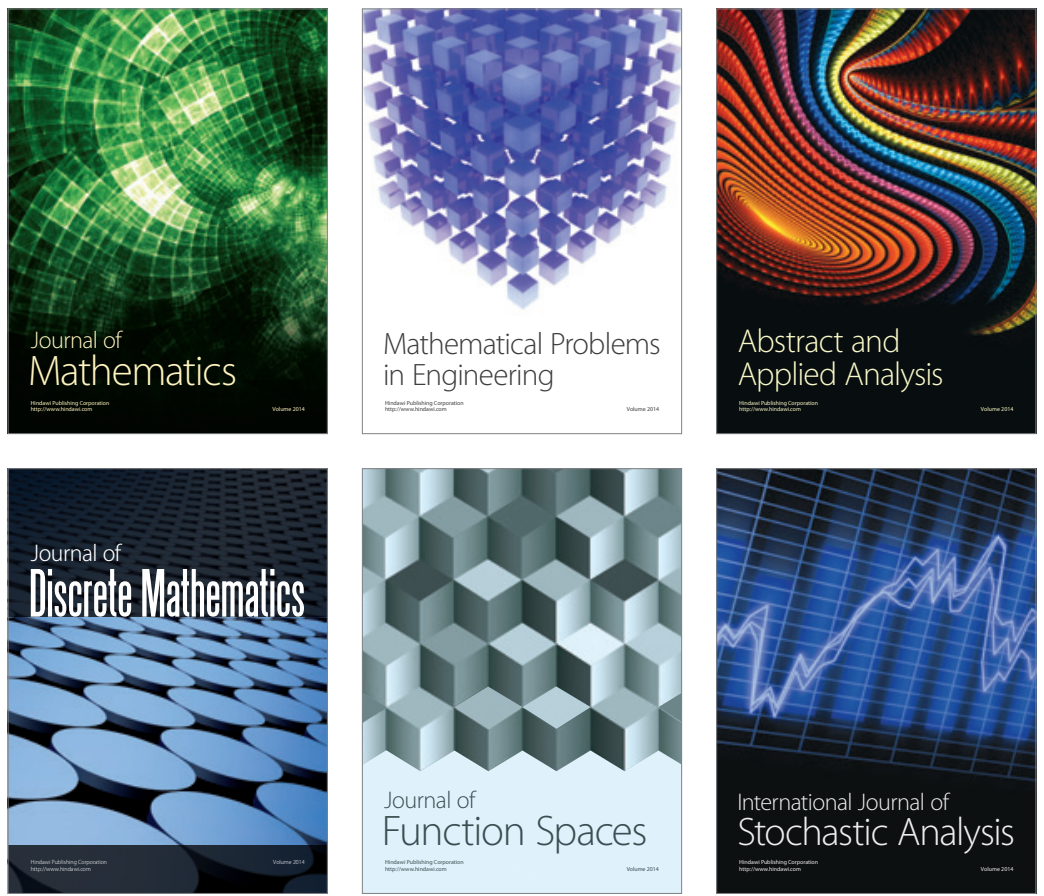

Journal of

Function Spaces

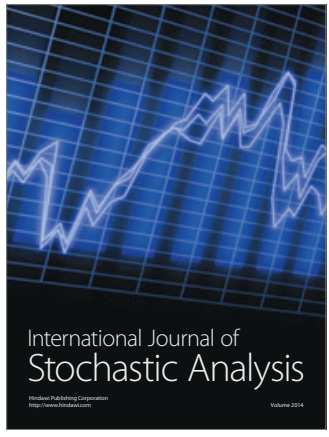

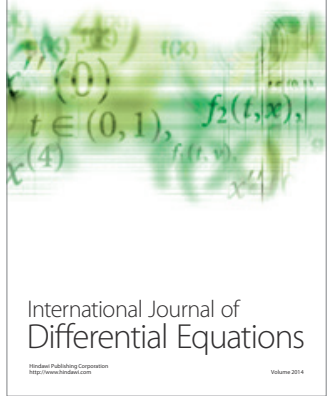
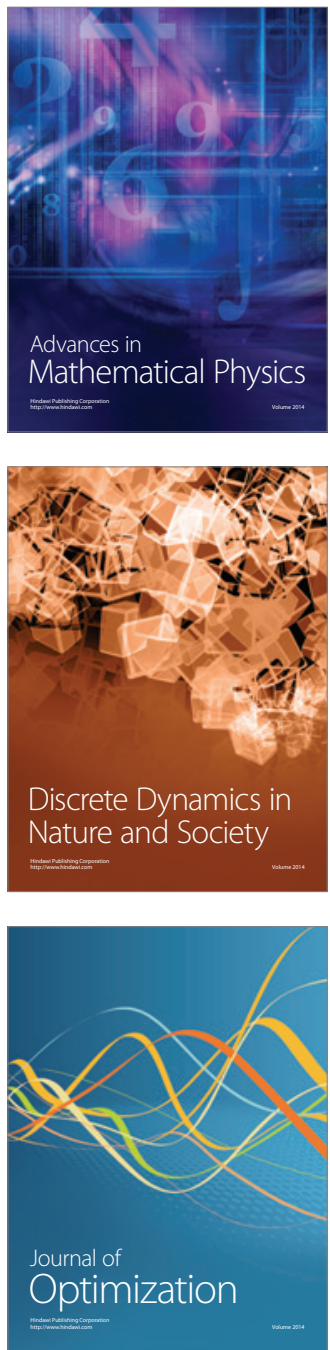